\title{
Autocuidado y calidad de vida en pacientes renales con tratamiento de hemodiálisis
}

\author{
Dr. Nigel Pascal Garcia Macías \\ nigelgarcia1@gmail.com \\ https://orcid.org/0000-0002-1594-6996 \\ Universidad Laica Eloy Alfaro de Manabí \\ Dra. Andrea Sthefania Racines Delgado \\ sthefaniaracines.m02@gmail.com \\ https://orcid.org/0000-0002-6947-3696 \\ Universidad Laica Eloy Alfaro de Manabí \\ Dr. Rafael Alejandro Peñafiel Cevallos \\ larvas01@hotmail.com \\ https://orcid.org/0000-0003-4766-1124 \\ Universidad Central Del Ecuador \\ Dra. Laleshka Nayarit Bravo Rodríguez \\ laleshkabravo@hotmail.com \\ https://orcid.org/0000-0001-8352-2987 \\ Universidad Técnica de Manabí
}

\section{RESUMEN}

El paciente con Insuficiencia Renal Crónica en tratamiento con hemodiálisis, enfrentan una serie de retos a nivel personal como problemas médicos, psicológicos y sociales, donde la complejidad de la misma aumenta con el tiempo y están en relación con las fases de la patología y los constantes procesos terapéuticos que alteran el bienestar biopsicosocial y la calidad de vida. El propósito del estudio fue analizar el nivel de autocuidado y la calidad de vida de los pacientes renales con tratamiento de hemodiálisis. Se realizó un estudio descriptivo, analítico no experimental, para la recolección de los datos se utilizó instrumentos como encuesta destinada a identificar el nivel de conocimiento sobre el autocuidado y calidad de vida de 50 pacientes que acuden a la clínica Metrodialisis; y entrevista a especialistas para conocer y analizar cómo influye la enfermedad en la calidad de vida de los pacientes. La investigación permitió identificar que los pacientes tienen conocimientos de autocuidado en la nutrición, administración de fármacos y cuidados en accesos vasculares, el dominio de salud física es poco limitada y el dominio psicológico reciben apoyo familiar al igual que indican que se sienten 
tranquilos, alegres y estables. En conclusión, el nivel de conocimientos y calidad de vida en el dominio físico y psicológico es significativo, porque realizan prácticas adecuadas de autocuidado que ayudan a conllevar de mejor manera la enfermedad y su tratamiento de hemodiálisis para mejorar su calidad de vida.

Palabras Claves: insuficiencia renal; fistula arteriovenosa; estilo de vida. 


\title{
Self-care and quality of life in kidney patients with hemodialysis treatment
}

\begin{abstract}
Patients who suffer from renal failure and are undergoing hemodialysis Treatment, face a series of challenge at a personal level such as medical, psychological and social problems, where the complexity of the pathology and the constant therapeutic processes altering their biopsychosocial well-being and quality of life. The objective was to analyze the level of self-care and quality of life renal patients with hemodialysis Treatment. It was carried out a transversal study of quali-qualititative, descriptive, non-experimental analytical type, 50 nephropathy patients who come to the radial clinic were studied, instruments such as the survey were applied to ident the level of knowledge about selfcare and quality of life of thepatients. The data provided indicate that de patients in hemodialysis Treatment suffer a negative impact in which their lifestyle Will be affected and Will have radical change, whichcan be evidenced in physical, psychological, social and economic repercussions, so patientsWill have to adapted to the various changes that Will have in their daily lives, the level of knowledge that is provided by health personal has significant value, since this implies that they can perform self-care practice that allow them to cope White the disease and adapt to treatment.
\end{abstract}

Keywords: renal failure; arteriovenous fistula; lifestyle

Artículo recibido: 10 Agosto. 2021 Aceptado para publicación: 07. Setiembre. 2021

Correspondencia: nigelgarcia1@gmail.com Conflictos de Interés: Ninguna que declarar

\section{INTRODUCCIÓN}


La Insuficiencia Renal Crónica (IRC) se define en términos científicos como la pérdida progresiva, permanente e irreversible de la tasa de filtración glomerular en un tiempo variable, que puede tomar años, expresada por la reducción del aclaramiento de creatinina estimado $<60 \mathrm{ml} / \mathrm{min} / 1.73 \mathrm{~m}$. (Sociedad Española de Geriatría y Gerontología, 2006) En la actualidad la enfermedad constituye un problema de salud pública a nivel mundial debido a la prevalencia e incidencia de casos que van en aumento en la población.

Este incremento se debe a varios factores como es la diabetes mellitus, hipertensión arterial, afecciones propias del riñón y estilo de vida de las personas lo cual conlleva a que personas jóvenes sean más susceptibles a ser diagnosticadas con esta enfermedad ( Veletanga, 2016). Es muy frecuenteque un paciente presente hipertensión arterial y diabetes durante el proceso de la patología, por lo que el daño sobre los riñones se incrementa. El control adecuado de la hipertensión arterial y la diabetes enlentece la progresión de la enfermedad renal crónica y disminuye el riesgo cardiovascular.

Por otra parte, la Organización Mundial de la Salud (OMS) señala que al menos un 10\% de la población a nivel mundial padece de insuficiencia renal, especialmente en aquellos paísesde ingresos medios y bajos. Esta enfermedad puede ser asintomática, aparece en etapa más avanzada en consecuencia, el paciente deberá seguir un tratamiento invasivo y de mayor costo como es la diálisis, sin embargo, en otros casos puede resultar en el trasplante de riñón (OPS, 2015)

A nivel de Latinoamérica las incidencias son similares, no obstante, la mayoría de los pacientes que padecen de esta patología no tienen acceso a una solución total del problema, es decir un trasplante renal, sino que son sometidos a hemodiálisis. De acuerdo a las estadísticas, durante las dos últimas décadas se ha presentado un desconcertante aumento decifras de mortalidad. Referente a los datos disponibles, la tasa de incidencia de ingresos a hemodiálisis para el año 2017 fue más alta en Puerto Rico con 1.500 pacientes, seguida por Chile 944 pacientes; Uruguay 765 pacientes; Argentina 659 pacientes; Brasil 490 pacientes y en Ecuador con 464 pacientes; la tasa más baja de incidencia fue para Costa Rica con 283 pacientes ( Silva Tobar, 2016). La tasa de prevalencia indica que son 650 pacientes por cada millón de habitantes, es decir que anualmente existe un aumento del $10 \%$.

En el Ecuador existen cerca de 10.000 personas que padecen insuficiencia renal y que necesitan terapia dialítica. Según el último reporte del Instituto Nacional de Estadística y 
Censo hasta el 2016 se contabilizaron 9.635 personas que padecen de insuficiencia renal. Porel contrario, las estadísticas se acrecientan, no por el aumento en la prevalencia de la enfermedad, sino más bien porque existe un limitado acceso al sistema de salud lo que impideque el paciente cumpla con su tratamiento ( Lucas-Choez, Cevallos-Carrión, Quiroz-Figueroa, \& Piguave-Figueroa, 2021).

Haciendo énfasis en la hemodiálisis se determina que es una de las terapias más utilizadas, pero provoca afectaciones como fatiga, cansancio, palidez, pérdida de peso, entre otras que indudablemente afectan la calidad de vida del paciente teniendo que adaptarse a un nuevo estilo de vida, y a ser dependiente de este procedimiento para lograr su supervivencia. Por tanto, la demanda de atención integral deberá abordar no sólo la propia enfermedad, sinotambién el funcionamiento físico, psicológico y social que permitan minimizar las complicaciones del paciente.

Este procedimiento puede variar de un paciente a otro, haciendo que la adaptación en ocasiones no sea fácil, ya que tiene consecuencias devastadoras que repercuten en su calidadde vida. La clave para mejorar este proceso es involucrar al cuidador, mismo que provocaráun impacto positivo dentro del estado emocional del paciente, permitiendo que en conjunto se superen una serie de retos biopsicosociales.

Por lo tanto, la calidad de vida en relación a la salud se define como sentimientos que refierenlas personas sobre sus expectativas y perspectivas en relación a la vida, donde incluyen sus metas, objetivos, valores, sueños y esperanzas (NMH Departamento de Nefrología de NMH, 2019). Los pacientes con insuficiencia renal al ser sometidos a terapias dialíticas como la hemodiálisis, tienen una modificación drástica ensus hábitos cotidianos, por lo que se altera su percepción de vida, generando sentimientos o expectativas a través de la falta de control del tiempo y de la vida, así como también temoresrelacionados con la muerte, tensión familiar y dependencia.

Por cada año se suman mil personas nefrópatas en la lista de las estadísticas, es decir que cada tres personas mueren diariamente esperando la donación de un órgano, 1.700 anualmente presentan algún tipo de enfermedad renal y de ellas alrededor de 500 necesitan trasplante renal. Aproximadamente el 35\% de los pacientes con problema renal reciben atención, pero 1000 de estos pacientes son beneficiados con el programa del Ministerio de Salud Pública (MSP), tanto en los hospitales de especialidades, como en las diferentes unidades de diálisis (Rodríguez Vidal, Merino Escobar, \& Castro Salas, 
2009).

Es importante señalar que en investigaciones realizadas por el Hospital de Especialidades Carlos Andrade Marín (HCAM) de la ciudad de Quito, a la Insuficiencia Renal Crónica se laconsiderada como una de las patologías de alto costo por generar gran impacto a nivel económico, y personal para la vida del paciente y su familia, incluida la repercusión laboral;se estima que su manejo ha comprometido en los últimos años el $2 \%$ del gasto de salud del país, y el 4\% de gasto en seguridad social ( Aragón \& Zapata, 2016). Teniendo en cuenta la magnitud de esta enfermedad, el autocuidado es otro de los puntos importantes a abordar. Está fundamentado en la teoría de Dorotea Orem que lo define comoel conjunto de acciones e interacciones que realiza la persona para controlar los factores internos y externos, que pueden comprometer su vida o su desarrollo posterior ( Prado Solar, González Reguera, Paz Gómez, \& Romero Borges, 2014).

Es importante entonces que tanto los pacientes, como los cuidadores conozcan el procedimiento, tratamiento y su autocuidado, evitando futuras complicaciones por lo que esnecesaria su educación continua. Si las personas adquieren una educación oportuna adecuadae individualizada, integrando diversos aspectos, se espera que ellos se hagan responsables del cuidado de su propia salud.

Es trascendental recordar que la percepción de necesidad de cuidado es única de cada persona, al igual que las acciones y el modo en que cada uno las realiza para cuidar de sí mismo porque están determinadas por los hábitos y las costumbres, es decir, por las condiciones sociales. La calidad de vida de estos pacientes depende de varias dimensiones e indicadores los cualesson físicos, mentales, emocionales, desarrollo personal, relación interpersonal, inclusión social, materiales, autodeterminación y derechos, los cuales permiten al paciente adaptarse asu enfermedad, al tratamiento y a los efectos de uno y de otro.

Para algunos investigadores el rol del personal de enfermería es fundamental, además del cuidado durante la hemodiálisis, ya que deberá adoptarse a la recomendación de trabajos realizados en otros países donde se integre al paciente en programas de deportes controladosy moderados, en la cual el paciente puede realizar ejercicios de las zonas del cuerpo que no está en contacto con la fístula arteriovenosa y el catéter venoso; así como otras propuestas derelajación (Organizacion Mundial de la Salud, 2020).

Es importante que el enfermero establezca una mayor interrelación con el paciente y a su 
vezcon el cuidador, con la finalidad de que puedan expresar sus emociones (miedos, estrés, tristeza, etc.), este proceso contribuye a brindarle una mejor contención al paciente y mejorala confianza entre el personal de salud, paciente y cuidador. En la medida que la confianza se refuerce el paciente podrá cumplir con las recomendaciones de autocuidado y terapéuticas.

En nuestro país la atención de salud brinda a los pacientes un plan de mejora de sus estilos de vida, implementando actividades interdisciplinarias que en consecuencia mejoren la calidad de vida de quienes son sometidos al tratamiento de hemodiálisis. La dieta, actividadfísica, descanso, sexualidad y control emocional son algunos de los aspectos que los pacientescon insuficiencia renal tienen que afrontar, adoptando medidas que conllevan al bienestar, locual no resulta tan fácil de llevar a cabalidad porque tolera su esfuerzo físico y mental.

Este proceso es diferente para aquellos pacientes de edad avanzada que padecen de IRC, puesdiversas investigaciones señalan que la enfermedad desencadena cambios en varias dimensiones de su vida, por síndromes geriátricos como la fragilidad que es altamente prevalente en la población renal, y está relacionado a una mayor vulnerabilidad de situacionesde estrés, lo que provoca debilidad, problemas de movilidad y equilibrio. Así también aumenta con la edad, y el estadio de la enfermedad renal provocando ciertas condiciones como la pérdida de energía y proteínas, inflamación, anemia, acidosis y trastornos hormonales. Se estima que existe de un 40 a $70 \%$ de prevalencia de fragilidad en el ancianocon IRC que requiere terapia dialítica. Por lo tanto, este síndrome debe ser detectado a tiempopara iniciar el tratamiento dialítico en el paciente con IRC debido al nivel elevado de morbimortalidad (Valladares Benitez \& Bonilla Quevedo, 2017)

El profesional de enfermería tiene un papel principal en el paciente renales lo que indica unaexcelente relación con el paciente y el cuidador responsable del mismo, permitiendo fortalecer el compromiso de autocuidado del usuario favoreciendo el bienestar físico y psicológico del mismo, de igual manera se encuentra en la capacidad de elaborar un plan decuidados que con lleve a la mejoría de la persona afectada, claro que este plan se realizará 


\section{DESARROLLO}

A continuación, se detallan los análisis de las tablas de las encuestas aplicadas a los pacientesrenales con tratamiento de hemodiálisis.

Tabla 1: Edad y Género

\begin{tabular}{|l|l|c|c|c|}
\hline Orden & \multicolumn{1}{|c|}{ Alternativas } & F & \% & Total \\
\hline & Masculino & 32 & 64,00 & \\
\hline Género & \multicolumn{2}{|c|}{} & 100,00 \\
\hline & Femenino & 18 & 36,00 & \\
\hline Edad & 45 a 54 años & 25 & 50,00 & \\
\hline & 55 a 63 años & 6 & 12,00 & 100,00 \\
\hline & 64 años o más & 19 & 38,00 & \\
\hline
\end{tabular}

Fuente: Pacientes con insuficiencia renal en tratamiento de hemodiálisis

En la tabla 1 se evidencia que el género masculino con edad de 45 a 54 años de edad presentaninsuficiencia renal y se encuentran con tratamiento de hemodiálisis, sobre esto la Dra. Lourdes Murillo especialista en nefrología y trasplante renal, manifestó que en investigaciones actuales indican que el sexo masculino tiene mayor probabilidad de desarrollar esta enfermedad renal de igual manera manifestó que la edad predominante dentrode las unidades de diálisis se encuentran pacientes con edad de 40 años en adelante.

Tabla 2: Relación de las variables en los pacientes con hemodiálisis

\begin{tabular}{|l|l|c|c|c|}
\hline Orden & \multicolumn{1}{|c|}{ Alternativas } & F & \% & Total \\
\hline \multirow{2}{*}{ Tiempo en } & Menos de un año & 0 & 0,00 & \\
hemodiálisis & Un año & 13 & 26,00 & \\
& Dos años & 15 & 30,00 & 100,00 \\
& Más de dos años & 22 & 44,00 & \\
Frecuencia de & 1 vez por semana & 6 & 12,00 & \\
tratamiento & 2 veces por semana & 27 & 54,00 & \\
& 3 veces por semana & 17 & 34,00 & 100,00 \\
& 3 veces por semana & 0 & 0,00 & \\
\hline
\end{tabular}

Fuente: Pacientes con insuficiencia renal en tratamiento de hemodiálisis

Los pacientes encuestados tienen más de dos años con tratamiento de hemodiálisis el mismoque se lo realizan 2 veces por semana. La especialista nos comenta la pauta más frecuente dentro de la hemodiálisis es de 4 horas y generalmente los pacientes pueden 
necesitar de 2 veces a la semana el procedimiento, claro que la frecuencia y la duración dependerá principalmente del grado de limpieza que el organismo requiera, presencia de infecciones y el estado nutricional del paciente, cabe recalcar que el tratamiento se suspenderá cuando exista la posibilidad de un trasplante y el mismo sea exitoso.

\section{Tabla 3: Cumplimiento nutricional en los pacientes renales}

Fuente: Población con insuficiencia renal en tratamiento de hemodiálisis

\begin{tabular}{|l|l|c|c|}
\hline \multicolumn{1}{|c|}{ Orden } & \multicolumn{1}{|c|}{ Alternativas } & F & \% \\
\hline a) & Siempre & 28 & $\mathbf{5 6 , 0 0}$ \\
\hline b) & A veces & 22 & $\mathbf{4 4 , 0 0}$ \\
\hline b) & Nunca & 0 & $\mathbf{0 , 0 0}$ \\
\hline Total & & $\mathbf{5 0}$ & $\mathbf{1 0 0 , 0 0}$ \\
\hline
\end{tabular}

Acerca del estado nutricional en los pacientes con insuficiencia renal influirá en el progresode la enfermedad y se asocia con el incremento en el riesgo cardiovascular, en estos pacientesse deberá considerar como un punto importante dentro de su tratamiento que el tanto el paciente y el cuidador mantengan hábitos alimentarios y estilos de vida adecuados los cualesson factores que desarrollaran la progresión al daño renal, por lo que la asesoría dietética quesea brindada deberá a estar orientada a prevenir sobrepeso y la desnutrición. argumentar bieneste análisis e incluir un estudio que haga referencia con el estado nutricional

El cuidado de la alimentación mediante el cumplimiento de las recomendaciones nutricionales juega un rol importante a lo largo del proceso de la insuficiencia renal, debido principalmente a la modificación y los requerimientos en macronutrientes y micronutrientesconforme evolucione la enfermedad, y en qué estadio se encuentre la enfermedad, cabe recalcar que en esto pacientes se tendrá en cuenta el peso ideal mas no el real debido a que este último puede sobreestimar la sobrehidratación, obesidad o malnutrición (Valladares Benitez \& Bonilla Quevedo, 2017).

Tabla 4: Indicador de conocimientos sobre una dieta equilibrada 


\begin{tabular}{|l|l|c|c|}
\hline Orden & \multicolumn{1}{|c|}{ Alternativas } & F & $\%$ \\
\hline a) & $\begin{array}{l}\text { Guineo, Alimentos con alto contenido de sal, enlatados, } \\
\text { granos, verde - maduro, líquidos }\end{array}$ & 0 & $\mathbf{0 , 0 0}$ \\
\hline b) & Toda clase de alimentos, pero en cantidades mínimas & 42 & $\mathbf{8 4 , 0 0}$ \\
\hline b) & Puedo consumir toda clase de alimentos sin restricciones & 8 & $\mathbf{1 6 , 0 0}$ \\
\hline Total & & $\mathbf{5 0}$ & $\mathbf{1 0 0 , 0 0}$ \\
\hline
\end{tabular}

Fuente: Pacientes con insuficiencia renal en tratamiento de hemodiálisis

Los pacientes con insuficiencia renal indicaron que pueden consumir toda clase de alimentos, pero en cantidades mínimas, lo cual puede ser un conocimiento acertado debido a que la dietavaría mucho y dependerá del estadio en que se encuentre el paciente como lo comentó la Dra.Murillo, que el plan dietético deberá ser individualizado y siempre varía de unpaciente a otro, el sodio en esto pacientes tendrá que ser limitado debido a que el consumo de este además de elevar la presión arterial, retiene líquidos, un paciente con hemodiálisis requiere una cantidad mayor de proteína es importante que se escoja las proteínas que tenganpoca cantidad de grasa y carbohidratos que les ayuda como fuente principal de glucosa en elorganismo, se debe limitar dependiendo del nivel que se encuentre, en cuanto al fósforo tiene una principalfunción dentro del organismo que es la formación de los huesos y dientes, en el paciente renalse encontrará totalmente limitado, de una manera general la dieta de estos pacientes estará enlimitar las cantidades de potasio, sal y fósforo.

Tabla 5: Cantidad de agua

\begin{tabular}{|l|l|c|c|}
\hline \multicolumn{1}{|c|}{ Orden } & \multicolumn{1}{|c|}{ Alternativas } & F & \% \\
\hline a) & Ninguno, no puedo beber líquidos & 16 & $\mathbf{3 2 , 0 0}$ \\
\hline b) & Puedo beber abundante líquido & 4 & $\mathbf{8 , 0 0}$ \\
\hline b) & Mínimo un vaso de agua & 30 & $\mathbf{6 0 , 0 0}$ \\
\hline Total & & $\mathbf{5 0}$ & $\mathbf{1 0 0 , 0 0}$ \\
\hline
\end{tabular}

Fuente: Pacientes con insuficiencia renal en tratamiento de hemodiálisis

En pacientes con insuficiencia renal crónica que se encuentren en tratamiento de hemodiálisisla cantidad de orina que se produce indica el requerimiento de líquido que necesitará el paciente, de manera general no es aconsejable beber más de 1-1/2 litro de líquidos, al contrario de la insuficiencia renal leve, normalmente la cantidad de orina en 
estos pacientes se conserva o puede llegar a ser superior a la normal por lo que la cantidad de líquido en estospacientes no está restringida.

El control de líquidos es un aspecto más importante dentro del manejo de los pacientes renales, el volumen de líquido recomendado dependerá de la función renal, la presión arterialy de la diuresis, estos se restringen según los factores ya mencionados con la finalidad de lograr un balance entre lo que se consumen y se orina. Al no funcionar los riñones los pacientes acumulan gran cantidad de líquido ingerido y eliminan sólo cuando se someten al tratamiento En caso de que se presente oliguria o anuria la ingesta se encuentra restringida.

La Dra. Murillo comenta que las vacunas dentro de estos pacientes son importantes, los pacientes se encuentran inmunosuprimidos, es decir que su sistema inmunológico será de respuesta lenta y a medida que la enfermedad avanza genera que el organismo no genere anticuerpos por ejemplo un paciente que se vacune en hemodiálisis no hace anticuerpos y para que tenga protección para la hepatitis B tendrá que vacunarse 5 veces para estar protegido, las vacunas recomendadas son influenza, neumococo, hepatitis B o que tengan elesquema de vacunación completa.

\section{Tabla 6: Dimensiones de la calidad de vida}

\begin{tabular}{|l|l|c|c|}
\hline \multicolumn{1}{|c|}{ Orden } & \multicolumn{1}{|c|}{ Alternativas } & F & \% \\
\hline a) & Bienestar físico & 7 & $\mathbf{1 4 , 0 0}$ \\
\hline b) & Bienestar psicológico o emocional & 19 & $\mathbf{3 8 , 0 0}$ \\
\hline c) & Autocuidado independiente & 2 & $\mathbf{4 , 0 0}$ \\
\hline d) & Satisfacción espiritual & 0 & $\mathbf{0 , 0 0}$ \\
\hline e) & Calidad de vida global & 22 & $\mathbf{4 4 , 0 0}$ \\
\hline Total & & $\mathbf{5 0}$ & $\mathbf{1 0 0 , 0 0}$ \\
\hline
\end{tabular}

Fuente: Pacientes con insuficiencia renal en tratamiento de hemodiálisis

Como se puede presenciar en la tabla, la mayoría de la población estudiada indica que la calidad de vida global se encuentra afectada, en relación a este punto nuestros profesionalesnos manifiestan que cuando los pacientes enfrentan o se enteran de que padecen de insuficiencia renal se hacen una serie de preguntas, es un periodo de tiempo en la cual afectaindudablemente al estilo de vida diaria. El manejo de estos pacientes deberá ser de manera integral entre los profesionales de salud y por grupos de autoayuda en la que ellos puedan expresar sus sentimientos, miedos y dudas acerca de esta 
enfermedad lo que nos permite quelos pacientes se adapten al tratamiento.

En la experiencia del Dr. Osorio nos cuentan que la insuficiencia renal crónica genera un impacto y cambia el estilo de vida de manera irreversible, debido a que tiene repercusiones físicas, psicológicas, sociales y económicas que no afecta solo a la vida de los pacientes, sinotambién al cuidador, pero a pesar de que se enfrentan todos los días al procedimiento se observa que la mayoría de los pacientes se adaptan y lo afrontan de manera positiva graciasal apoyo que brinda el equipo integral de salud y el más importante la familia.

\section{Tabla 7: Autocuidado}

\begin{tabular}{|c|l|c|c|}
\hline Orden & \multicolumn{1}{|c|}{ Alternativas } & F & \% \\
\hline a) & $\begin{array}{l}\text { Guineo, Alimentos con alto contenido de sal, enlatados, } \\
\text { granos, verde - maduro, líquidos }\end{array}$ & $\mathbf{0}$ & $\mathbf{0 , 0 0}$ \\
\hline b) & Toda clase de alimentos, pero en cantidades mínimas & $\mathbf{4 2}$ & $\mathbf{8 4 , 0 0}$ \\
\hline c) & Puedo consumir toda clase de alimentos sin restricciones & $\mathbf{8}$ & $\mathbf{1 6 , 0 0}$ \\
\hline Total & & $\mathbf{5 0}$ & $\mathbf{1 0 0 , 0 0}$ \\
\hline
\end{tabular}

Fuente: Pacientes con insuficiencia renal en tratamiento de hemodiálisis

De acuerdo a los conocimientos que tienen los pacientes en el cuidado de la fístula indican que siguen las recomendaciones brindadas por parte del personal de salud, Tanto el Dr. Osorio como la Dra. Murillo definen que la fístula es la unión de una arteria con una vena lacual permite que el flujo sanguíneo sea mayor, toda fístula o injerto es un acceso vascular que permite conectar al paciente con la máquina de hemodiálisis, es importantísimo que el paciente logre tener un cuidado óptimo de la fístula en su hogar como palpar y observar constantemente la zona donde se encuentre la fístula, evitar usar ropa ajustada, pulseras o reloj en la extremidad que se encuentra la fístula, no permitir que le tomen la presión o extraigan sangre en el brazo donde se encuentre la fístula, evitar vendaje compresivos dondese encuentren la fístula, no colocar cremas o ungüentos donde se encuentre la fístula al menosque esté indicado hacerlo, algo que es de suma importancia evitar dormir sobre la zona dondese encuentre. Todas estas recomendaciones son brindadas tanto como al paciente y cuidadoresto ayudará que la vida de la fístula sea alargada.

\section{Tabla 8: Limitaciones en actividades}




\begin{tabular}{|l|l|c|c|}
\hline \multicolumn{1}{|c|}{ Orden } & \multicolumn{1}{|c|}{ Alternativas } & F & \% \\
\hline a) & Muy limitada & 21 & $\mathbf{4 2 , 0 0}$ \\
\hline b) & Poco limitada & 26 & $\mathbf{5 2 , 0 0}$ \\
\hline c) & No limitada & 3 & $\mathbf{6 , 0 0}$ \\
\hline Total & & $\mathbf{5 0}$ & $\mathbf{1 0 0 , 0 0}$ \\
\hline
\end{tabular}

Fuente: Pacientes con insuficiencia renal en tratamiento de hemodiálisis

La tabla nos indica que la actividad física es poco limitada, en referencia a esta pregunta nuestros profesionales nos indican que el ejercicio en los pacientes con hemodiálisis es muyimportante ya que ayudará a prevenir los riesgos de caídas en estos pacientes mejorando su estilo de vida, al hacer ejercicio el cuerpo libera sustancias que ayudan a mejoran la autoestima con la cual los pacientes podrán tener independencia y esto les permitirá adaptarseal tratamiento y disfrutar de la vida, el ejercicio físico que realicen estos pacientes mejora lacalidad de vida además que hace que el periodo de sus sesiones sea mucho más corta.

Tabla 9: Bienestar psicológico

\begin{tabular}{|l|l|c|c|c|}
\hline \multicolumn{1}{|c|}{ Orden } & \multicolumn{1}{|c|}{ Alternativas } & F & \% & Total \\
\hline \multirow{2}{*}{ Red de Apoyo } & Si & 35 & $\mathbf{7 0 , 0 0}$ & $\mathbf{1 0 0 , 0 0}$ \\
& No & $\mathbf{1 5}$ & $\mathbf{3 0 , 0 0}$ & \\
\hline \multirow{2}{*}{ Estado anímico } & Tranquilo, alegre y estable & $\mathbf{2 9}$ & $\mathbf{5 8 , 0 0}$ & $\mathbf{1 0 0 , 0 0}$ \\
& Angustiado, triste y preocupado & $\mathbf{2 1}$ & $\mathbf{4 2 , 0 0}$ & \\
\hline
\end{tabular}

Fuente: Pacientes con insuficiencia renal en tratamiento de hemodiálisis

En la parte del apoyo familiar el $70 \%$ de los encuestados manifestaron que reciben apoyo familiar al igual que indican que se sienten tranquilos, alegres y estables. De acuerdo a estoslos entrevistados dicen que la actuación en todo el proceso que llevará el paciente presenta cambios, tanto en las actividades de la vida diaria como en el estado anímico.

$\mathrm{Al}$ momento que el paciente se entera que padece de insuficiencia renal y que tiene que someterse a tratamiento de hemodiálisis, le produce una serie de inquietudes y expectativas sobre su evolución y mejora personal en su tratamiento esto les crea un entorno de ansiedadque repercutirá negativamente en su bienestar biopsicosocial. Porque incluir a la familia, pues este pequeño paso crea un impacto positivo debido a que sentirá el apoyo en esta etapadifícil de su vida, no solo a la hora del tratamiento de hemodiálisis, 
sino también en su hogardonde tendrá que cumplir con la dieta adecuada a su enfermedad, la toma de medicación correcta y sobre todo la estabilidad emocional que tendrá que ser tratada en conjunto a su familia, debido a que estos pacientes presentaran cambios de humor e incluso de carácter quetendrán que ver con la nueva situación de salud.

\section{CONCLUSIONES}

- La capacidad de autocuidado en los pacientes con insuficiencia renal y tratamiento de hemodiálisis es significativa, lo que indica que las habilidades y los conocimientos para realizar acciones de autocuidado podrán ejecutar actividades de la vida cotidiana permitiendoadaptarse y afrontar a la enfermedad.

- En cuanto a la calidad de vida en el dominio físico los pacientes con tratamiento de hemodiálisis presentan pocas limitaciones y realizan ejercicio físico de moderada intensidadcomo caminar, levantar brazos, extender y flexionar las piernas, apretar la pelota con la manopor lo que esto les ayuda a mejorar significativamente la capacidad funcional además de aumentar la fuerza muscular, prevenir la pérdida de masa muscular, incrementar la función cardiovascular y optimizar la supervivencia al reducir el riesgo cardiovascular.

- La calidad de vida en el dominio psicológico de los pacientes en tratamiento de hemodiálisisse evidencio que reciben el apoyo de parte de la familia lo cual es importante para que los pacientes mantengan sentimientos positivos, debido a que esto les permite acoplarse al tratamiento y al estilo de vida que conlleva durante todo el proceso de la enfermedad.

\section{REFERENCIAS BILIOGRAFICAS}

Aguilar Chalacán, M. F., Vela Benalcazar, C., Recalde, J. L., \& Negrete, R. (2021). Mesenteritis esclerosante: diagnóstico y tratamiento. Scielo, 16. doi:10.30944/20117582.707

Akram, S., Pardi, D., Schaffner, J., \& Smyrk, T. (2007). Mesenteritis esclerosante: características clínicas, tratamiento y resultado en noventa y dos pacientes. $\begin{array}{llll}\text { Gastroenterología } & \text { clínica } & y & \text { hepatología, }\end{array}$ doi:https://doi.org/10.1016/j.cgh.2007.02.032

Aragón, E., \& Zapata, G. (01 de 2016). Evaluación de la calidad de vida en pacientes con enfermedad renal crónica en tratamiento sustitutivo. Cambios, 16. doi:https://doi.org/10.36015/cambios.v15.n1.2016.179 
Emory , T., Monihan, J. M., \& Carr , N. (2017). Mesenteritis esclerosante, paniculitis mesentérica y lipodistrofia mesentérica: ¿una sola entidad? PubMed, 13. doi:10.1097 / 00000478-199704000-00004

Labalde Martíneza, M., Guijarro, J., Pacheco Martínez, P., Fernández Escuder, B., Domingo Asenjo, T., Jiménez de los Galanes, S., . . Perea Fernández, F. (2016). Mesenteritis esclerosante como causa excepcional de dolor abdominal. Elsevier, 12. doi:10.1016/j.gastrohep.2010.12.004

Lucas-Choez, M. M., Cevallos-Carrión, D. A., Quiroz-Figueroa, M. S., \& PiguaveFigueroa, T. J. (2021). Autocuidado y calidad de vida en pacientes renales con tratamiento de hemodiálisis. Polo del Conocimiento, 12. Recuperado el 01 de 08 de 2021, de file:///C:/Users/Usuario/Downloads/2292-12364-4-PB.pdf

Poniachik, J., \& Smok S, G. (2000). Mesenteritis retráctil Comunicación de cuatro casos. Scielo, 16. doi:http://dx.doi.org/10.4067/S0034-98872000001100010

Prado Solar, L. A., González Reguera, M., Paz Gómez, N., \& Romero Borges, K. (2014). La teoría Déficit de autocuidado: Dorothea Orem punto de partida para calidad en la atención. Scielo, 6. doi:http://scielo.sld.cu/scielo.php?script=sci_arttext\&pid=S1684$18242014000600004 \& \operatorname{lng}=\mathrm{es} \& \ln \mathrm{ln}=\mathrm{es}$.

Sharma, P., Yadav, S., Marie Needham, C., \& Feuerstadt, P. (02 de 2017). Mesenteritis esclerosante: una revisión sistemática de 192 casos. Springer, 10. doi:https://doi.org/10.1007/s12328-017-0716-5

Silva Tobar, S. D. (2016). HEMODIÁLISIS: ANTECEDENTES HISTÓRICOS, SU EPIDEMIOLOGÍA EN LATIONOAMÉRICA Y PERSPECTIVAS PARA EL ECUADOR. UNIANDES EPISTEME: Revista de Ciencia, Tecnología e Innovación., 3(1), 18.

Veletanga, J. (03 de 2016). Edicion medica. Recuperado el 04 de 08 de 2021, de https://www.edicionmedica.ec/secciones/salud-publica/en-ecuador-cerca-de-10mil-personas-necesitan-di-lisis-87408

Delgado Plasencia , 1., Rodríguez Ballester , López Tomassetti , F., A Hernández Morales, A., \& Carrillo Pallarés . (2007). Paniculitis mesentérica: experiencia en nuestro centro. PubMed, 14. doi:10.4321 / s1130-01082007000500010

Navarro, S., \& Rivas Rivas, M. (2018). Mesenteritis esclerosante "aparente enfermedad 
maligna": alternativa diagnóstica ante la presencia de dolor, ascitis y suboclusión intestinal. Sociedad Andaluza de Patologia DIgestiva, 11. doi:This article is available from https://www.sapd.es/revista/2018/41/2/07

NMH Departamento de Nefrología de NMH. (Noviembre de 2019). Insuficiencia Renal: Elección del Tratamiento. Northwestern Medicine Hospital, 11. Recuperado el 05 de 08 de 2021, de file:///C:/Users/Usuario/Downloads/northwestern-medicineinsuficiencia-renal-eleccion-del-tratamiento-kidney-failure-treatments.pdf

OPS. (10 de 03 de 2015). Organizacion Panamericana de la Salud. Recuperado el 04 de 08 de 2021 , de https://www3.paho.org/hq/index.php?option=com_content\&view=article\&id=10 542:2015-opsoms-sociedad-latinoamericana-nefrologia-enfermedad-renalmejorar-tratamiento\&Itemid=1926\&lang=es

Organizacion Mundial de la Salud. (2020). COVID-19; Coronavirus; Ética en Investigación; Bioética. Organizacion Mundial de la Salud, Estados Unidos. Recuperado el 13 de 03 de 2021, de https://iris.paho.org/handle/10665.2/52142

Rodríguez Vidal, M., Merino Escobar, M., \& Castro Salas, M. (2009). VALORACIÓN PSICOMÉTRICA DE LOS COMPONENTES FÍSICOS (CSF) Y MENTALES (CSM) DEL SF-36 EN PACIENTES INSUFICIENTES RENALES CRÓNICOS EN TRATAMIENTO CON HEMODIÁLISIS. Ciencia y Enfermeria, 15. Recuperado el 05 de 08 de 2021, de https://www.redalyc.org/pdf/3704/370441798009.pdf

Sánchez, A., Albertini, C., Cárdenas, K., Pérez, G., \& Bécker, J. (2002). Mesentiritis esclerosante. Presentación de un caso y revisión de la literatura. Bibliografia Latinoamericana, 15.

Sociedad Española de Geriatría y Gerontología. (2006). Tratado de geriatria para residentes. En L. Z. Rubenstein. Madrid, España: Copyright. Recuperado el 04 de 08 de 2021, de https://www.segg.es/tratadogeriatria/pdf/s3505\%2000_primeras.pdf

Valladares Benitez, B. M., \& Bonilla Quevedo, G. L. (2017). "Supervivencia y desigualdad social en adultos mayores con Enfermedad Renal Crónica estadio 5 en tratamiento de hemodiálisis usuarios del centro CLINEF NORTE en el periodo comprendido de 01 enero de 2007 al 31 de diciembre de 2017". Quito. 
Recuperado el 05 de 08 de 2021, de http://repositorio.puce.edu.ec/bitstream/handle/22000/16727/SUPERVIVENCIA \%20Y\%20DESIGUALDAD\%20SOCIAL\%20EN\%20ADULTOS\%20MAYOR ES\%20EN\%20HEMODIALISIS.pdf?sequence=1\&isAllowed=y 